\title{
Dynamics in artifact ecologies
}

\author{
Susanne Bødker \& Clemens Nylandsted Klokmose \\ Department of Computer Science \\ Aarhus University \\ Aabogade 34, 8200 Aarhus N, Denmark \\ \{bodker, clemens\}@cs.au.dk
}

\begin{abstract}
We increasingly interact with multiple interactive artifacts with overlapping capabilities during our daily activities. It has previously been shown that the use of an interactive artifact cannot be understood in isolation, but artifacts must be understood as part of an artifact ecology, where artifacts influence the use of others. Understanding this interplay becomes more and more essential for interaction design as our artifact ecologies grow. This paper continues a recent discourse on artifact ecologies. Through interviews with iPhone users, we demonstrate that relationships between artifacts in artifact ecologies cannot be understood as static, instead they evolve dynamically over time. We provide activity theory-based concepts to explain these dynamics.
\end{abstract}

\section{Author Keywords}

Artifact ecology, activity theory, dynamics

\section{ACM Classification Keywords}

H.5.m. Information interfaces and presentation (e.g., HCI): Miscellaneous.

\section{INTRODUCTION}

Our technological habits are changing rapidly and we are witnessing a surge of what the late Steve Jobs and his followers refer to as "post-PC" devices. People do computing on the couch, on the beach, in the train, and it is not uncommon for people to own multiple devices with highly overlapping functionality, from desktop or laptop computers to pads, tablets and smartphones.

By acquiring these post-PC devices, we create or extend our ecology of artifacts: the artifacts that we own, have access to and use, as defined by [9]. However, when acquiring such a device people have only vague ideas of what it actually is and how they are going to use it-they might end up using it in a different way than expected, and begin to use their other artifacts in different ways as well.

Recently researchers have begun articulating the interplay between the heterogeneous interactive artifacts people use $[9,13,14]$. However, the focus has mainly been on a static, here and now, image. In this paper we discuss the dynamics of artifact ecologies when new artifacts are introduced. We provide a nuanced interpretation of artifact ecologies, as something highly dynamic and in constant interplay with users' web of activities, and their expectations of future use. This leads to a set of concepts rooted in activity theory and dialectical thinking that help articulate these dynamics.

Our discussions are exemplified with interviews of twelve iPhone users on their appropriation and use of iPhones. These interviews provide insights into how their artifact ecology is influenced by the introduction of a new device.

\section{BACKGROUND}

The word ecology is borrowed from Gibson [8]. In the Gibsonean sense, our (visual) perception is shaped by our physical ecology and cannot be understood in isolation. The ecology of a subject is the part of the physical world that it interacts with to realize its life. Following Gibson's definition of ecologies, Jung et al. [9] define a person's ecology of artifacts as the artifacts "... that a person owns, has access to, and uses".

With the personal computer, a radically new kind of artifact was introduced into our artifact ecologies. This artifact substituted more and more traditional artifacts to a point where many occupations, especially those of knowledge workers, could be almost completely realized through this single artifact. Hence, in the optics of HCI, the artifact ecology of users has been simple; we only had to understand one artifact, the PC. During the last decade, non- $\mathrm{PC}^{1}$ interactive artifacts have become more powerful and more commonplace. The ecology of interactive artifacts has gone from being simple (a single personal computer) to being complex (music players, gaming consoles, smartphones, PCs, entertainment systems, interactive walls etc.) [11].

However, until five years ago, the most widely used nonPC interactive devices were special purpose devices such as cell-phones and music players. There were few other devices that had the same general-purpose nature as the PC, and even fewer of them were mass-market consumer

Pre-print of paper for NordiCHI 2012

\footnotetext{
${ }^{1}$ When we speak of non-PC artifacts, we mean personal computing devices that are neither a desktop nor a laptop computer.
} 
devices $^{2}$. This changed when Apple released the iPhone in 2007, and the following year the App Store opened and let users download new functionality for their mobile device directly on the device. Since the introduction of the iPhone, smart-phones with similar functionality and extendibility have been introduced, and yet another form factor for computers, the pad, has been introduced. It doesn't end here; wall and table displays are slowly becoming affordable, and could be the next type of mass-market non-PC.

This development means that people are acquiring interactive artifacts that are different from their personal computers, at the same time as they are general-purpose artifacts. In the following we explore the effects of introducing such an interactive artifact on the overall artifact ecology.

\section{RELATED WORK}

Jung et al. [9] introduced the concept of artifact ecologies. Based on the assumption that artifacts cannot be fully understood individually, they empirically explored the relationships between interactive artifacts in people's personal life. Their approach was to make people map their artifact ecologies and describe the relationships between included artifacts. This approach clearly showed how the respondents distributed their activities across different artifacts, and how artifacts had different roles, influenced by the capabilities of the other artifacts in the ecology. This work was followed up with a tool for people to map their ecology of artifacts [13]. However, the approach provides a static image of an artifact ecology. We are interested in conceptualizing the dynamics of artifact ecologies when a person acquires a new artifact, especially when the artifact isn't a special purpose device, but has capabilities that may be shared with other devices in the artifact ecology.

Carroll et al. [5] studied the appropriation of WAP phones among young people. They developed a model for appropriation or "the way that users evaluate and adopt, adapt and integrate a technology into their everyday practices" based on three "levels", which are: first encounters, exploration and long-term integration into everyday practices. Our study below has led to a somewhat similar focus on states, yet we start off differently: Where users, in [5], were given the cell-phones to use, our users purchased their own phones. Hence they have considered why they wanted the phone, which is our starting point. Accordingly, we will return to the differences and similarities between our states and the three levels of [5].

Karapanos et al. [10] conducted an "over time study" where they followed six iPhone users for the first five weeks from purchase. They analyzed the data through a framework for experience and appropriation as three experience phases in

${ }^{2}$ One example is the Nokia 9000 Communicator from 1996 that was an early general-purpose mobile with some success with business users. sequence (Orientation, Incorporation, Identification), driven by the forces of Familiarity, Functional Dependency and Emotional Judgment, respectively. This is pivoting around expectations, formed by anticipation. [10] points out that the iPhone is a success both as an object of desire that sells, and as something that is appreciated in long-term use.

Bødker \& Christiansen [2] share our empirical foundation, but their analytical agenda is quite different. They expanded on [10]'s and [5]'s scope towards users' social setting rather than individual orientation, and looked for patterns in the webs of activities where users are involved rather than unidirectional expansion. They defined 'appropriation' as an individual-in-context movement of complementary nature, where learning to operate develops as does the context of use. They pointed out how appropriation requires acting of the user, at the same time as the resistance is both socio-cultural and physical. Mature users of iPhones have decisive moments, where they recognized that they had made the iPhone their own. The iPhone becomes an instrument of quite idiosyncratic activity such as poetry reading or Italian language studies. Such new uses are important and under development in the mature use situation. Underlying the ongoing development of the iPhone-in-use, simplicity and usability remained stable qualities, according to [2].

In this paper, we do not focus on the appropriation of an artifact per se, but rather on how the appropriation of an artifact influences and is influenced by the overall use of the artifact ecology that it becomes part of.

\section{THEORETICAL FRAMING}

The aim of the paper is to better understand what happens when a new technology is infused into an artifact ecologyunderstanding the singular artifact in the complex arrangement of people, organization, other mediating technologies, etc. Where e.g. the presentation of artifact ecologies in [9] is ad-hoc, individual and static, Activity Theory, which we use as our theoretical framing, offers a focus on the dynamics of such complex arrangements of people and mediating technologies.

According to activity theory, users' shared capacities and experiences are not only based on individual acting and learning in the world: People act in cultural situations where they get to share practices, and the role of the more capable peers is important in this [15]. Characteristic to the dialectical thinking of activity theory, each individual, who is part of such practice, continues this practice. When we look in further detail at the relationship between the user and the artifact, it is on this background: There is no user without other users who share their experiences with artifacts and materials, understanding, etc.

With our activity theoretical background, we argue in [3] that the artifact ecology of an individual is defined by the set of artifacts applied to realize the activities of the individual. Hence, the artifact ecology is shaped by a web- 
of-activities, where the same artifact can be part of multiple activities, or artifacts can be substituted [4] for each other to realize the same activity. The web-of-activities of an individual is highly dynamic; therefore with the above definition, the artifact ecology of an individual is highly dynamic.

If we think of the artifact ecologies we surround ourselves with, they most often consist of multiple artifacts built for similar purposes but with slight variations and no clear delineation of when to use which artifact-e.g. a fountain pen vs. a ballpoint pen. The specific choice of artifact that the user may make is situated and depending both on the material conditions of the activity and on the specifically intended outcome, such as when a user chooses to read a document on a PC versus on a smartphone. The artifact ecology is in continuous development. Artifacts come and go; they can break and be replaced, or their function becomes obsolete due to changing circumstances, activities or because of newly acquired skills.

Every artifact contains the germ of a new practice and remnants of old practice. Bardram \& Bertelsen [1] use the concept initial familiarity to describe the phenomenon of how new artifacts or artifacts used in a new use situation can trigger reapplication of previously learned actions. Initial familiarity can be constituted in interface design through playing on simple affordances or e.g. interface metaphors. According to Gal'perin [7] the scaffolding for the further development of use is constituted in the mediating artifact as well as socially e.g. through more capable peers. According to Engeström [6] the tension between the given new (the artifact being explored in this case) and the expected new as what ultimately drives change. This dialectics is an example of the dialectical method of reasoning that aims to understand things concretely in all their movement, change and interconnection, with their opposite and contradictory sides in unity [3].

In this paper we draw on a recent elaboration of activity theoretical HCI leading to the Human-Artifact Model [3]-a means to analyze the use of artifacts in context of use and their artifact ecology. The analytical scheme of the HumanArtifact Model (Figure 1) combines analyses of human experiences and artifacts, and addresses the tensions between human skills and capacity on the one hand, and the action possibilities and affordances offered by the artifact on the other [3]. This is done on three levels reflecting the activity hierarchy: activity, action and operation. These levels provide three sets of analytical glasses, each of which focuses on an important aspect of human activity: Motivation (by asking why?), goal-orientation (by asking what?) and operation (by asking how?). The focus of an analysis with the Human-Artifact Model is on tensions between the experiences of an individual and the assumptions of use embodied in the artifact on all three levels of activity.

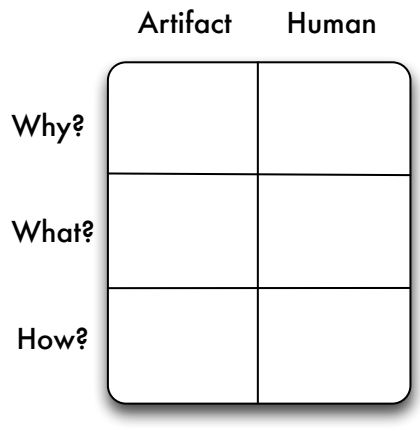

Figure 1 Overall structure of the Human-Artifact Model

\section{THE CASE AND METHOD}

To leverage the discussion of the dynamics in artifact ecologies, we analyze interviews from a study executed by the first author where twelve iPhone users were interviewed about their appropriation and use of iPhones. These interviews provide insight in the effect a new general-purpose interactive artifact has on users' existing artifact ecology and their use thereof.

12 interviews were carried out with iPhone users in the age range from 19 to 62 , three men and a woman at 19-23 years of age, a man and two women around 60, and the remaining interviewees spread in their 30s and 40s (three men and two women). In addition, five were re-interviewed after a year.

At the time of the interviews, four of the interviewees had owned their phones for 2-3 months, while at the other extreme two had had iPhones before they were officially introduced in their country. Three people owned iPhones previous to their current phone and an additional three owned an iPod Touch. All had other cell-phones previous to the iPhone (but only one had another brand smartphone before). One had been a very long-term Mac user, two were Mac users before purchasing their iPhone, and four purchased Macs after acquiring their iPhone. Four were current PC users. They all volunteered, or got volunteered by friends, through a request on Facebook.

The interviews consisted of semi-structured questions to look back on the motivations for acquiring an iPhone, their artifact ecology, the early experiences of use as well as their current use and expectations for the future. They lasted between 25 and 45 minutes and were recorded, transcribed and translated. The transcriptions were coded and used to identify themes and patterns [12]. The interviews were used as the basis for [2], as well as the current paper.

The coded interview fragments were annotated with their relationships to the levels and sides of the Human-Artifact Model. A hypothesis emerged that there were three rather well defined stages in the development of the artifact ecologies of our interviewees. In the following we present the interviews in this structure of three stages, followed by an analysis conceptualizing these stages to three states, which the artifact ecologies of the interviewees' iterate through. 


\section{INTO THE ARTIFACT ECOLOGY}

We have transformed our readings of the material to narratives, supplemented with quotes in order to present our results. The italicized words come directly out of the translated material. Since our focus is on the role of the iPhone as artifact making its travels into the artifact ecologies of the users, we have been less concerned with representativity and more with the complex varieties of mediation that the interviewees talk about. Nonetheless, we see obvious similarities and differences in themes and patterns that we hope get conveyed to the readers.

\section{Anticipating the new-great expectations}

The interviewees were all asked to tell us about their reasoning for acquiring an iPhone and their expectations towards the new device, when looking back at this after some months.

Among our interviewees there are three quite distinctly different types of background (with one exception of an interviewee who had previously owned another smartphone, the interviewees spread quite equally in these three categories):

1. Users with no previous experience of iPods, iPhones or other Apple products in their artifact ecology

2. Users with Apple products in their background artifact ecology, but no specific smartphone or iPod Touch experience

3. Users previously using an iPod Touch as part of their artifact ecology.

Users who had no previous experience with iPods, iPhones or other Apple products were primarily looking for a cool telephone. Everybody had previously owned cell-phones and was experienced in making phone calls as well as in texting on cell-phones of various kinds, which hence belonged to their artifact ecology. Their expectations were inspired by their network of friends and family, and even by the blogs they were reading. The main expectation was to get a new cool telephone that would also support text messaging. Some of the interviewees saw the iPhone as slightly big. None of them were immediately expecting to substitute e.g. their current camera or music player with the new iPhone, nor were they interested in the access to web browsing, Facebook and such. Accordingly, they saw their iPhone as something that would substitute their previous cell-phone in their artifact ecology, but nothing more.

Interviewees with Apple background experience consisted of seasoned Mac users, who came to the iPhone more with an expectation of robustness and technical integration. They wanted to supplement their computer and artifact ecology at large with an iPhone that was expected to be robust like the Mac, and integrate with this artifact ecology. This included one interviewee who had specifically acquired the iPhone together with a small group of colleagues to co-manage contact information for their students and share calendars. (Q1): "I got the phone through work. We wanted a shared calendar for four of us, and perhaps later for the entire school. We put phone numbers of all students on the phones, and that has turned out to be really useful for me as study counselor. The shared calendar was less successful, but in other ways the iPhone is highly addictive."

While this interviewee had a rather functional motivation for the purchase, others in this category mentioned that they were early movers regarding technological gadgets and that they shared this passion with friends face-to-face, on Facebook, etc. The calendar functionality as well as the robustness were mentioned as expectations from this group. These interviewees, however, shared with many other interviewees the expectation that the iPhone would be well designed and addictive. This was about moving to the $21^{\text {st }}$ century. Steve Job's world!-all quite non-functional expectations.

People who had previous experiences with the iPod Touch very much expected something like iPod Touch but with a phone. A robust, all-in-one device. They shared with the other groups the passion, and addiction that lies outside a specific need or problem in their current artifact ecology, at the same time as they had a particular motive of purchasing an iPhone: that of reducing the number of different artifacts that they had to carry (Q2): "I was very pleased with my dear old Nokia, but it would only text and make phone calls. My friends bought me an iPod Touch for my birthday, and I got hooked. I knew I needed an iPhone. Getting an easy to use device that would do everything!"

Applying the Human-Artifact Model to summarize this stage, what characterizes the expectations in general for the new iPhone in interviewees' artifact ecologies, is that they are mostly related to non-functional aspects of the phone. Instead their desire and expectations for the device are driven by a desire to own something cool, or even be cool, together with their community. Hence most of the action happens on the 'why' level of the Human-Artifact Model. Yet, the more specific the actual background experiences of the users, the more specific was their expectations to how their artifact ecology would change. These expectations were med differently in the actual meeting with the iPhone as we discuss below.

\section{Exploration-stirring up}

When actually getting hands on the phone, the interviewees describe a highly exploratory stage. They talk about exploring the app store, pursuing recommendations from media or friends, etc. Many of the interviewees talk about how they explore free apps of all sorts only to abandon them again shortly after. Games, music instruments and similar such apps are particularly prone to be tried and thrown away. Among the more durable apps that people start using at this stage are maps, train and bus schedules, radio, calen- 
dar apps and apps that are particular useful to their school or studies. The iPhone itself gets explored and opens the doors to an artifact ecology including the App Store as such and the many apps available.

For some, the iPhone, as it was given, was not entirely living up to the expectations in two significant ways: First of all, these were severe problems of composing text messages. These problems were long lasting and consisted of several elements: People found the spelling correction annoying and not nearly as efficient as what they were used to with their Nokia and SonyEricsson background. The mere idea that you have to cancel corrections rather than approving them added to this. Some complained about the fact that you are given no tactile feedback when typing because everything happens through the soft keyboard. They were quite literally used to texting without looking, in their past artifact ecology.

Set-up problems were several. One interviewee did not think she was getting enough out of her iPhone because there was no manual which she had expected there to be (Q3): "I don't have time to immerse myself in the possibilities. I miss a manual. I don't mind reading manuals with my legs up."

Another interviewee couldn't get started to use the iPhone because his computer had crashed, and he had not been told that he needed that to get started with the iPhone. Apple has now changed this, but at the time, you needed an artifact ecology with a computer to start using an iPhone, and this was very unexpected to the interviewee. Seen through the lens of the the Human-Artifact Model, these start-up problems seem like clashes between what the iPhone actually offered and the expectations of the users, based on the background of their, then, current artifact ecology, more than actual problems with setting up the iPhone as such.

At this point in time, many of the interviewees described that they still used artifacts such as separate calendar, camera, music player, etc., from their artifact ecology. Even iPods are mentioned as being used initially in parallel with the iPhone.

Nonetheless, the interviewees started using the web browser, reading news, following the weather through apps or the link to the national weather forecast. They listened to music and podcasts (and in some instances acquired help from their spouses or friends to start with). They downloaded games to be used by themselves or children in their environment. With a couple of exceptions, Facebook was high on the list of apps that people started using when they were on the move, e.g. in the car, or on the train. Once people realized the potential of being on-line on the move, email also moved to the iPhone platform from the laptop when on the move. It seems that single, specific apps, that people perceived as useful, helped make the transition from simply a cool telephone to something more general that took a new role in their artifact ecology: For some this app was Facebook, for others e.g. a news reader, the app from their fitness center or a weather app.

The narrow social networks, around spouses, close family or friends were used to learn new things at this stage. In addition, some of the interviewees read popular media, and others more specialized media such as Slashdot, all of which became parts of their artifact ecologies. Spouses worked together on configuration, and friends, relatives and colleagues-one-on-one or small groups-explored the set-up their iPhones when they met (Q4): "My newest app? 'Radio P3', because I wanted to show to my Mom that you could listen to the radio." Weekends with a circle of friends, the lunch table at work, family parties, camps with colleagues and pupils, became the webs-of-activities where the iPhone was explored, tried out, and where the flashing of the iPhone as such was a ticket to belong, be cool, etc. (Q5): "70 percent of my closest girlfriends have an iPhone. I was on a girl-weekend, and I believe we were five out of eight with iPhones. We sit and talk about apps."

The iPhone was explored 'together with' the laptop as a remote control. People worked to synchronize with Google calendars, and with a shared address list, all part of the artifact ecology.

At this stage, interviewees describe how routines and rituals started to develop, involving the iPhone as well as other artifacts such as the computer: Routines developed for what was done on the computer versus the iPhone when at home, out, at work or other places, e.g. a parents' house. The iPhone was used for everything-calendar, music, cameraand the number of other artifacts surrounding the iPhone diminished.

One interviewee describes how she developed a wake-up ritual involving several apps in her artifact ecology (Q6): "I read email, watch TV2 and DR news in the morning. I sort of look through the phone in the morning (SMS, email, news, Facebook), right when I'm waking up. Perhaps earlier you had a clock-radio, now I wake up to the cellphone." Another interviewee talks about a moment where he realized how much the iPhone could do for him, changing his web-of-activities (Q7): "One day I was downtown waiting for somebody. While I waited I decided to book my hairdresser on-line. I found a document about our tutor schedule in 'First Class' and checked my schedule and homework in 'Lectio'. Before I knew, I was good to go!"

Both of these examples also illustrate that the interviewees had achieved a certain mastery of routines that were more general than the singular apps, similarly to how mastery is described in [7]. Both the use of the artifact ecologies and their webs-of-activities changed with this mastery.

Once interviewees crossed the barrier where they no longer perceived of their iPhone as a smart phone (or a substitute cell-phone), they no longer mention that the iPhone is big. In terms of the Human-Artifact Model, the new human 
motivation/'why' changes the 'how' of the artifact. The interviewees became regular users of some specific apps that were now part of their artifact ecology, and they knew that they could find and explore more, together with their friends when they meet them, or alone, with the App Store. Some of them found the exploration as such less interesting and relevant, perhaps because their mastery had become sufficient to move their attention away from the iPhone as such towards the activities it might mediated, the purposes it may fulfill.

\section{Equilibrium, still changeable}

After a very exploratory use of the iPhone, the interviewees describe how the iPhone became an everyday artifact that was used in conjunction with their other artifacts as part of their artifact ecologies in everyday webs-of-activities.

Music was played on a combination of media: CDs, MP3 players, computers, stereos and the iPhone. A couple of heavy music listeners still had old iPods full of music, one interviewee had no stereo but chose rather randomly between playing music from the computer and the iPhone, etc. Several interviewees used apps for maps and train and bus schedules, and to a large extent they didn't actually use these apps as part of their artifact ecology, rather they knew that they could be found in the App Store if needed, and hence included in the artifact ecology for a period of time. Generally people seemed to stop or limit their exploration of the App Store, and accordingly they also used fewer of the free apps that they used to explore. They were confident that something could be found in the App Store if needed.

The network of people with whom the iPhone was in shared focus similarly diminished, as the iPhone seems to gain more of a personal role to its users (as is developed extensively in [2]).

Various kinds of permanent and temporary "division of work" with computers, in the artifact ecology, were seen in the interviews. These include going online from the iPhone only in specific locations, or in particular situations. Actually for many interviewees the computer was the leanforward mode, doing "work", while the iPhone was often used in lean-back mode-idling, background check, etc. in addition to filling a role when away from home. (Q8): "I read e-mail on the computer if I can. The keyboard is better, and there is multitasking. But it is good to know that those things can be done on the iPhone. I have decided only to access Facebook from the iPhone. That's because it used to distract me and take too much of my time. Now I have better control over that, also since the Facebook app gives a different kind of access to Facebook."

Several of the interviewees mastered updating the software to the extent that it was not a conscious concern for them. How they did it varied. A couple of interviewees barely ever plugged their iPhone into their computer and their iTunes library, while at the other extreme some did this almost on a daily basis. Mastering one's travel without pre- planning and reading email from multiple devices are other examples of routines that were mastered at a high level, supported by the iPhone, the App Store and the underlying email software as part of the artifact ecology.

In contrast to this, the breakdowns and trouble with writing text messages continue, to the complaint of many interviewees, when asked what is the worst part of the iPhone (Q9): "The worst? That it writes 'St' every time I write 'at'!" This problem stayed with them, event though is seems to play a less dominant role in their everyday use. The iPhone as a telephone moved to the background, and other uses took dominance in the artifact ecology. Texting and phoning were critical uses nonetheless.

The iPhone at this stage was finding a role beyond simple routines. Examples are reading poetry in boring school lessons (see further in [2]), or reading texts in Italian whenever possible. This latter interviewee kept several Italian dictionaries and translators on her iPhone, to have them ready to hand. A third interviewee had become slightly addicted to composing music while sitting in cafés, new elements to his artifact ecology (Q10): "I bought 'iMachine' you can sit and make some beats. It has excellent samples, the people who made this are really pros. Sometimes when I sit in a café-it does not happen that often-and I get a beat into my head, I can try it out. Then an hour and a half has passed! Scary!"

A very different, but similarly specific kind of new use was when one of the interviewees talks about creating a hub-onwheels for his carpool. Every morning they simply took turns setting up one of their iPhones as wireless hub from which everybody in the car could work, a new role for the iPhone in the artifact ecology, and a more extended artifact ecology all together.

With these developments of the artifact ecology, the iPhone helped shape entirely new activities with new motives to the interviewees. This seems to be a matter of total use, not singular apps as contrast to the early use of the iPhone.

At this stage, the iPhone found a role in the artifact ecology, despite a few recurrent problems, in particular that of texting. Evidently there was a strong wish for an improvement with respect to texting. At the same time the interviewees expressed various new needs that basically came out of the current use. One interviewee had a long-lasting wish to make his own cookbook app. In the first interview he already mentioned this, and in the second interview this had taken more realistic forms, since he had realized that there was a lot of work in this that he couldn't do himself. Nonetheless, the dream lived on. Several interviewees in the last round mention the iPad as an alternative artifact, which few of them had actually used. (Q11): "I would probably pay for [e]books, but it [the iPhone] is too small, so you'll probably need an iPad". One of the interviewees goes on to talk about the use of iPhones and iPads to support education at the school where he works. Both the 
iPad specifically, and the visions of developing own apps and supporting new forms of education, can be seen as possible triggers of new rounds of development.

Summarizing these interviews through the Human-Artifact Model points towards new mastered routines at the 'how' level, such as reading email on various devices depending on location, with recurring breakdowns of texting that nonetheless get a different role, and new uses, like reading poetry, i.e. new 'why' and 'what'.

With this tour through the interview material and its mentioning of artifact ecological matters, we turn to a deeper understanding of artifact ecologies and their possible dynamics.

\section{ANALYSIS}

Our aim of this paper is to argue that artifact ecologies are dynamic and evolving, and to address the mechanisms of these dynamics. The relationships between artifacts change over time. In the above interviews we see examples of how such changes happen. The interviews provide a unique peek into what happens when users get hands on a new device, they don't really know what will do for them. It was quite clear from the interviews that there are different states in appropriating the iPhone, and hence different states that the interviewees' artifact ecologies pass through. We refer to $a$ stable state, an excited state, and an unsatisfactory state of the artifact ecology (Figure 2). Analyzing the interviews through the lens of the artifact ecology is of course not the only way to explain results from the interviews, as can be seen in [2]. However, by using the interviews to understand the artifact ecologies as people talk about them, it has become clear that we need a non-static understanding of this phenomenon.

\section{The unsatisfactory state}

The unsatisfactory state is when the artifact ecology no longer lives up to the needs of the individual and requires change. In traditional activity theoretical terminology, there is a contradiction between the capabilities of the artifacts in the ecology, and the activities of the user. This is where we start out with our interviews; all of our interviewees were to some degree unsatisfied with the state of their artifact ecology in that they have decided to acquire an iPhone. There are different factors that can bring an artifact ecology into an unsatisfactory state. It might be new functional or instrumental requirements, e.g. wanting to have a calendar that is synchronized with co-workers while on the go. It can also be the desire to consolidate multiple artifacts as the case with wanting to have an iPod Touch containing a phone. It can be that the user wants to separate functionality out in multiple artifacts by moving the use of calendars from the PC to the phone. However, in the case of our interviewees, the artifact ecology was mostly in an unsatisfactory state due to non-functional factors: the desire to

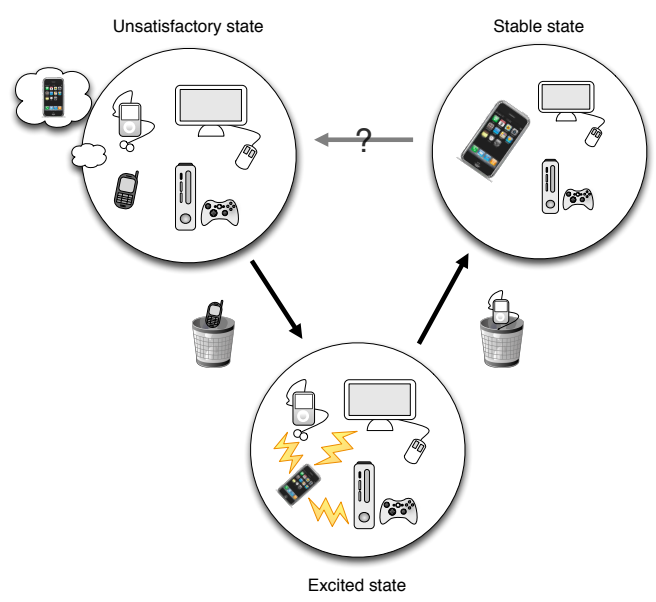

Figure 2. The states of the artifact ecology. In the unsatisfactory state the current artifact ecology no longer lives up to the users need, and a change is needed. In the excited state a new artifact is added to the ecology, perhaps replacing an old. This state is characterized by heavy exploration. The roles of the artifacts are reassessed. This leads to a stable state where the new and old artifacts have found their role-perhaps and old artifacts have been obsoleted. At some point the artifact ecology will become unsatisfactory again, and the process repeats.

acquire a new artifact as part of self-realization, or to live up to the social norm.

The step taken to remedy the unsatisfactory state is based on the expected new and assumptions shaped by past experience, research, and social influence from friends, family, co-workers and wider networks, e.g. Facebook and blogs. At the same time as this state is characterized as unsatisfactory, it is also a state of high hopes. High expectations of having functional problems solved, such as the shared calendar, or a better phone, or of integrating more functions, as with previous users of the iPod Touch. To a large extend these expectations at the 'what' level of Human-Artifact Model, addressing specific goals, seeking certain functions in the iPhone. As mentioned above this is combined with some rather vague ideas pertaining to the question of 'why?' of the Human-Artifact Model, like "moving into the $21^{\text {st }}$ Century."

At the 'how' level, it is fair to say that nobody expected problems with texting, yet they were evident for all interviewees. The previous users of iPod Touch describe a rather smooth transition when it comes to the handling of the iPhone except for this area that was new with the iPhone. For everybody, the expectations were about cool yet robust handling, but nobody mentioned specifically e.g. that they were looking forward to touch interaction compared to their previous keypad.

\section{Excited state}

An artifact ecology enters an excited state when a new artifact is added. A mix of old routines and explorations of the potentials of the new artifact characterize the use in this 
state, hence in our empirical examples we talk about exploration and stirring up the artifact ecology.

The exploration and perception of the new artifact is highly shaped by initial familiarity. For many, the fact that the iPhone is a phone for calling and texting, actually leads to some interesting breakdowns between expectations and the reality of the given new artifact. In the design of the iPhone, trade-offs have been made between its role as a phone e.g. for texting and its general-purpose touch-based user interface. In the beginning many of the users found the experience of texting inferior, compared to traditional cellphones. Yet later, when they started exploring the full capabilities of the artifact, this trade-off seems to have become more and more acceptable, even though operationally, the problem did not go away, and breakdowns were recurring even for very experienced users.

In the exited artifact ecology, games play a role in the initial exploration of the interaction possibilities, however the presence of games on the iPhone is often excused by the presence of children. Interviewees similarly explore a range of rather useless free apps, and through this they learn both actual handling and more about what they might expect from apps. Exploring the App Store as such, looking at new apps, recommended apps, and most popular apps moves this trial-and-error exploration up a level, from single apps to the notion of apps and the possibilities of apps as such. The support network as well as blogs and news support and focus this exploration.

Several interviewees report set-up problems, yet new routines start to develop. Many of these seem to be triggered by single apps such as weather or fitness apps, which fulfill some sort of specific purpose for the user. Their use becomes mastered separately.

The iPhone is used intermixed with or as substitution for other artifacts: camera, calendar, iPod. This division of work is partly based on location (home, on the move, at parents) and some applications move back and forth a lot: Facebook, mail, news. As part of this explorative stage, some of these applications, for some users, become mastered across devices, which means that Facebook is routinely done on the device that is most easily at hand, or best fulfilling the purpose at the particular moment. The decisions of which device to use, moves from being a 'what?' to being a 'how?'

As we presented earlier, rituals start to emerge, and in general the web-of-activities in which the iPhone is used, extends from phoning and texting, beyond web browsing, to specific apps. The motivation for having an iPhone changes from being a smart phone to other specific motives, answering the 'why?' Some activities slowly move from other devices to the iPhone, e.g. reading mail, checking Facebook or the weather, or listening to music.

The network of more capable peers (friends, colleagues, relatives) plays an extensive role for those who successfully move past the early exploration. Influence from more capable peers such as family or co-workers plays an even bigger role than individual exploration. While there is uncertainty about potentials of the use of the iPhone at this stage, some of the interviewees describe how they learn more about uses they don't want, e.g. not reading email over the summer, not replacing the big screen and keyboard for certain purposes, changing the relationship between the human being and the artifact as captured in the HumanArtifact Model.

\section{Stable state}

In the stable state, the artifacts of the artifact ecology have found their role. The web-of-activities is simpler and each activity, in which the iPhone is used, is clearly motivated-it has a clear answer to the 'why?': Italian study, poetry reading, hub-on-wheels. The interviewees have given up on some of their previous artifact ecology, and the use of 'other' cameras, paper calendars, etc. is less frequent.

The interviewees use several self-invented rules when it comes to handling their artifact ecology in relation to music, Facebook and mail, which get done across locations and devices. Some interviewees have rules for 'always' doing Facebook on the iPhone, or 'always' reading email on the computer, with exceptions when they are away for longer time. Others use the iPhone in lean-back situations like over morning coffee, in bed, or in front of the TV, while using the computer when being lean-forward and doing work. These are high-level routines captured by the 'how' level of the Human Artifact Model.

For both the well-defined activities and these device-crossing activities have a certain level of mastering. The stable artifact ecology means no or little trial and error, and accordingly one may answer the question of 'how?' with a combination of general routines that largely include both handling of the PC and the iPhone for the specific activity, e.g. mail, and some rather specific high-level operations pertaining to Italian study artifacts or the like.

The answer regarding 'what?' is simpler than before, because there are fewer, and less exploratory purposes of use. When acquiring the iPhone it was critical for users to be able to make calls. This remains a critical aspect, yet it steps into the background of other features. New critical aspects arise through use, changing the routines of the user, e.g. as regards poetry reading. Other routines involve e.g. relying on being able to look something up on the phone e.g. to travel, and therefore not looking it up before leaving the house. The community is less active in shaping the artifact ecology in this state.

A stable state does not mean that development in use has stopped. To some extent such development seems more goal-driven, i.e. driven by the 'what' level, as when interviewees know they can find new apps to use when they travel to new places. In addition, new needs arise, based on the experiences, and these needs may eventually trigger a 
new transition to a new unsatisfactory state-one of our interviewees begin to talk about the iPad as a more suitable device for reading eBooks than the iPhone. Hence, new needs are starting to develop, that will eventually bring the artifact ecology back in an unsatisfactory state, as illustrated in Figure 3.

\section{SUMMARY}

In the above, we have used the basics of activity theory and the Human-Artifact Model to address artifact ecology as concept with the example of artifact ecologies of iPhone users. This theoretical basis has helped us focus on artifact ecologies as dynamic, being tightly coupled to the web of activities in which the iPhone is used, to the user's relationship with his or her community of fellow users, both narrowly (friends and relatives) and more widely, e.g. through Facebook and blogs.

The Human-Artifact Model has helped us focus on the dialectics between the expected new (as developed in and through use) and the actual use as it unfolds in the meeting with the given artifact (the iPhone), the App Store, and the community of fellow users.

Figure 3 sketches how the tensions between the artifact and the capabilities of the users shift across the levels of activity. Motivational and functional expectations create tensions in the unsatisfactory state, while the excited state is characterized by tensions and development on all levels of activity due to exploration of the artifact. In the stable state, there is still some development, but mainly in regarding what and why, whereas how the artifact is used is mostly established. This development both originates in continued exploration of the artifact and changing needs and requirements.

The familiarity of Apple's design program, and in particular the resemblance with the iPod Touch, support many users in getting started. The lack of manual, however, may do the opposite. The many inexpensive Apps of the App Store, along with the strictly enforced UI guidelines, scaffold the exploration. Furthermore, we have illustrated how the artifact ecology needs to be understood in close connection with the human development of routines and motives. In other word is the artifact ecology not just a list of functionality, of 'what?', it cannot be understood without the 'why?' and the 'how?.'

Our hypothesis that there are three delineated stages seems to hold, and brings insight to the analysis, e.g. so that we appreciate the richness of the explorative, excited state at the same time as we understand the mechanisms, both in terms of human appropriation, and the constitution of the artifact ecology, that makes it calm down.

Even though we study the artifact ecology of the individual, the artifact ecology is less individually constituted, and more dynamic than that of [9].

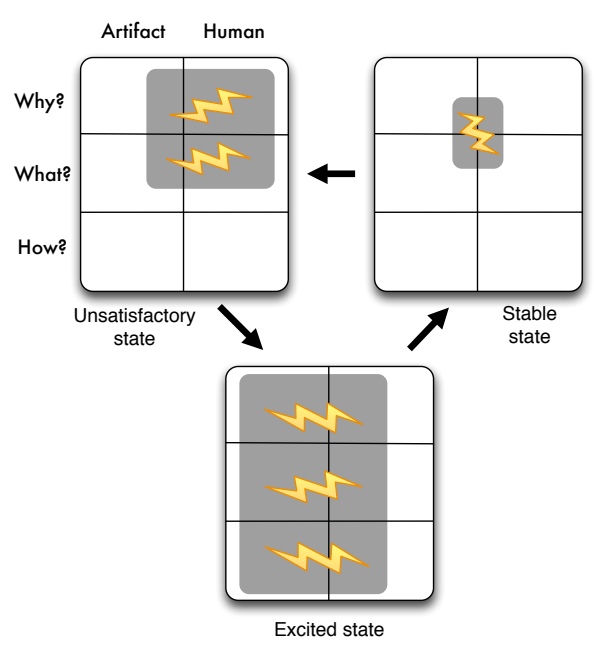

Figure 3. Focuses of tensions between the human and artifact in the three states

When bringing these details to the Human-Artifact Model, we add to Activity Theoretical HCI a further focus on details of the way in which experience of use develops and may be activated through design of artifacts, in particular non-PC artifacts to be applied in complex artifact ecologies. In particular we have focused the fundamental dialectics on the relationships between human capacities and action possibilities offered by the artifact on the three activity theoretical levels. As illustrated in Fig. 3, the stable state has the fewest and less critical tensions, in particular at the 'how?' level. Yet new needs develop in the artifact ecology that may eventually challenge the human-artifact relationship. New artifacts may be considered, and a new excited state occur.

\section{DISCUSSION}

We share with [5] the hypothesis of three states, or levels in their terms. The starting points for the two types of framings are different in that our analysis starts with the unsatisfactory state, with expectations and problems of users before buying. [5] gives WAP phones to users, and are in a different way confronted with prejudice more than expectations. Our second state comprises their two first levels and the analyses are in many ways quite parallel. Compared to the model of [10], expectations are not just part of the start of use. They exist throughout use as an understanding of where the user, perhaps together with other users, want to go next, as the fundamental tension that drives the development of the artifact ecology.

While it has not been at the core of our analysis to address what Apple has done right or wrong, it seems evident that the strict design guidelines and heavy control of the apps in the App Store, help users explore the action possibilities and build up routines, that are applicable across Apple platforms and apps. The free and inexpensive apps in the App Store serve well to help people explore. At the same time, our analyses of the unsatisfactory state seems to point to a 
curious issue, namely that Apple may initially have been slightly mistaken in terms of their conception of what the iPhone is/was to their users. The iPhone may have been designed to be an attachment to the Mac, rather than an independent phone, that could be bought and brought home, activated without a computer, but with a manual, and ultimately used for the things people (in our part of the World at least) do with cell-phones: texting! The texting problem remains. However, it becomes less important to the users as their attention to other uses of the iPhone grows.

We did not have access to the stable states of the artifact ecologies of the interviewees before they purchased their iPhone, and hence we know their motivation for purchasing their iPhone only in retrospect. With one exception, this motivation was, nonetheless, not a functional one. This points to the need for an artifact ecology framework that helps focus beyond function, such as the activity theoretical one.

What is really the artifact in the artifact ecology is an important challenge in our analysis: Sometimes it is the iPhone as a device that needs to be handled, sometimes it is the iPhone as an access point to the App Store, or a webbrowser, sometimes a specific app, and even other times it is the selection of apps on the iPhone that is in focus. This reflects the changing purposes of use that is central to activity theory, but also tells us that something additional may be needed to e.g. address the software systematically across technical platforms.

The framework points out that artifact ecologies are dynamics and we have demonstrated the power of such analyses in our case. To work further along these lines, longitudinal studies of the development of artifact ecologies are needed. We did re-interviews after a year, and found only little additional value from this. Hence, we suggest that future work must work on a different time scale, and that hence, getting back to the interviewees in five years would be more relevant.

\section{CONCLUSION}

Understanding the interplay between artifacts in users' artifact ecologies becomes increasingly important for interaction design as we distribute our activities across artifacts in idiosyncratic ways.

In this paper we have demonstrated that the use of artifact ecologies evolve dynamically over time. Based on a study of iPhone users, we conceptualize this dynamism as three states that artifact ecologies iterate through. We have learned that to fully grasp how artifact ecologies evolve, a study with a scope of one year is too short, and encourage longitudinal studies of artifact ecologies over five years or perhaps a decade.

\section{ACKNOWLEDGMENTS}

We thank the interviewees, as well as our collaborator, Ellen Christiansen, and Marianne Dammand Iversen, who worked on our language.

\section{REFERENCES}

1. Bardram, J. E. \& Bertelsen O. W. (1995). Supporting the Development of Transparent Interaction. In Blumenthal, B., Gornostaev, Y. \& Unger, C. (Eds.). EWHCI '95 Selected Papers. Springer. 79-90.

2. Bødker, S. \& Christiansen, E. (2012). Poetry in motion - appropriation of the world of Apps. In Proceedings of ECCE 2012, ACM Press (to appear).

3. Bødker, S. \& Klokmose, C. N. (2011) The HumanArtifact Model-An Activity Theoretical Approach to Artifact Ecologies. Human-Computer Interaction. 26, (4,): 315-371.

4. Brodersen, C., Bødker, S., and Klokmose, C. N. (2007) Ubiquitous Substitution. In Proceedings of INTERACT 2007. Springer. 179-192.

5. Carroll, J., Howard, S., Vetere, F., Peck, J., and Murphy, J. (2002). Just What Do the Youth of Today Want? Technology Appropriation by Young People. In HICSS'02, IEEE Computer Society. 1777-1785.

6. Engeström, Y. (1987). Learning by expansion. Helsinki: Orienta Konsultit.

7. Gal'perin, P.Y. (1969). Stages in the development of mental acts. In Cole, M. \& Maltzman, Y. Handbook of contemporary soviet psychology, Basic Books. 249-273.

8. Gibson, J.J. (1986). The ecological approach to visual perception. Erlbaum.

9. Jung, H., Stolterman, E., Ryan, W., Thompson, T., \& Siegel, M. (2008). Toward a framework for ecologies of artifacts: How are digital artifacts interconnected within a personal life? Proc. of the NordiCHI 2008. ACM Press. 201-210.

10. Karapanos, E., Zimmerman, J., Forlizzi, J. \& Martens, J.B. (2009). User experience over time: an initial framework. In Proc. of CHI '09. ACM. 729-738.

11. Klokmose, C. (2012) On Human-Computer Interaction in Complex Artefact Ecologies. PhD Dissertation. Aarhus University.

12. Kvale, S. (1994). InterViews. Hans Reithel

13. Ryan, W., Stolterman, E., Jung, H., Siegel, M., Thompson, T., and Hazlewood, W.R. (2009) Device ecology mapper: a tool for studying users' ecosystems of interactive artifacts. In Proc. of CHI '09. ACM. 43274332.

14. Schilit, B.N., Sengupta, U.(2004). Device ensembles. Computer, 37(12): 56- 64.

15. Vygotsky, L.S. (1978). Mind and society: The development of higher mental processes. Harvard. 